\title{
Patient safety and nurse managers' competencies IN DECISION MAKING RELATING TO RATIONING OF CARE THE STATE OF THE ART
}

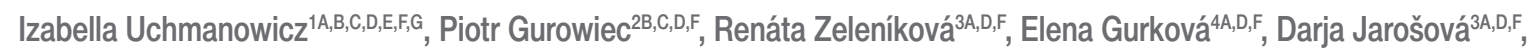
Agnieszka Zborowska ${ }^{1 \mathrm{~A}, \mathrm{~B}, \mathrm{E}, \mathrm{F}}$

${ }^{1}$ Faculty of Health Sciences, Wroclaw Medical University, Wroclaw, Poland ${ }^{2}$ Department of Nursing, Opole Medical School, Opole, Poland

${ }^{3}$ Department of Nursing and Midwifery, University of Ostrava, Ostrava, Czech Republic

${ }^{4}$ Department of Nursing, Palacký University Olomouc, Olomouc, Czech Republic

Authors' contribution:

A. Study design/planning $\bullet$ B. Data collection/entry $\bullet$ C. Data analysis/statistics $\bullet$ D. Data interpretation $\bullet$ E. Preparation of manuscript $\bullet$ F. Literature analysis/search $\bullet$ G. Funds collection

\author{
Address for correspondence: \\ Prof. Izabella Uchmanowicz \\ Department of Clinical Nursing \\ Wroclaw Medical University \\ 5 K. Bartla St., 51-618 Wrocław, Poland \\ e-mail: izabella.uchmanowicz@umed.wroc.pl \\ SUBMITTED: 15.03 .2020 \\ ACCEPTED: 24.04 .2020 \\ DOI: https://doi.org/10.5114/ppiel.2020.96091
}

\begin{abstract}
To prevent potential health- or life-threatening events during patient care at every stage of hospitalisation, all possible causes that could lead to adverse events should be recognised by the highly qualified medical staff who are responsible for patient safety.

The aim of this state-of-the-art paper is to summarise the issue of patient safety in the hospital and nurse managers' competencies relating to rationing of care, and to discuss implementation of the evidence-based practice as the key foundation for the development and knowledge management of nursing care.

Research evidence does not provide any innovative solutions to direct knowledge management in clinical settings. Therefore, we should implement innovative interventions to be developed and evaluated for the implementation of knowledge management. The fact is that a global lack of nurses leads to missed care, which is a common threat, and it manifests itself as a potentially dangerous medical error that deserves special attention.

The influence of various factors on knowledge management and the roles of senior nurses have not been well recognised or researched. Moreover, it was shown that practical mediations in order to implement knowledge management need to be refined and assessed. This is especially true in contemporary, challenging work environments with a lack of time for further professional development due to an increasing workload.

Key words: patient safety, nurse managers, competency, rationing, state of the art.
\end{abstract}

\section{INTRODUCTION}

Patient care is a planned process that includes a variety of actions to improve the patient's health condition. With the current development of medical technology, a human on the one hand can count on rapid therapeutic effects but on the other hand may be exposed to various types of health- or life-threatening events. To prevent this, all possible causes that could lead to the risk of adverse events should be known. Hospitals, to ensure the safety of patients at every stage of hospitalisation, i.e. during diagnosis, treatment, nursing, or rehabilitation, are required to guarantee the adequate level of service. The most important elements in this regard, along with infrastructure and equipment, are highly qualified medical staff who are aware of their responsibility for patient safety.

The aim of this state-of-the-art paper is to summarise the issue of patient safety in the hospital, nurse managers' competencies relating to rationing of care, and to discuss the implementation of evidence-based practice (EBP) as the key foundation for the development and knowledge management of nursing care.

\section{PATIENT SAFETY}

According to the Agency for Healthcare Research and Quality (AHRQ) definition from the Health and Safety Commission of Great Britain (HSCGB): "The safety culture of an organisation is the product of individual and group values, attitudes, perceptions, competencies, and patterns of behaviour that determine the commitment to, and the style and proficiency of, an organisation's health and safety management" [1].

Patient safety culture (PSC) has been defined as an organisation's commitment, style, and proficiency in safety management and is determined by 
the views, behaviours, and competencies of individuals and groups defined by Lee et al. (1996) in their definition of PSC. In order to assess PSC in different clinical settings, a range of different tools have been developed [2-4].

The World Health Organisation (WHO), the World Alliance on Patient Safety (WAPS), the Council of Europe, European patient groups, doctors, nurses, pharmacists, and hospitals are actively involved in ensuring the safety of patients. Close coordination, and effectively and consistently implemented policies in this area will contribute to improving the quality of health care and provision of patients with a high level of safety. The WHO's work in this field first began in 2004 with the inception of WAPS, an agenda that has steadily grown over time. In order to prevent patient harm on a global scale the WHO Patient Safety and Risk Management (PSRM) has been created. Its job is to oversee and improve innovations in patient safety and care as well as manage risks in health care [5].

The European Network for Patient Safety Project identified 19 different survey tools and other methods in use throughout the European Union (EU) member states until 2010 for measuring patient safety culture [6]. Among the validated tools that are widely used to assess the culture of patient safety by recognising the perception of health care providers is the Hospital Survey on Patient Safety Culture (HSOPS) and the Safety Attitudes Questionnaire (SAQ) [7].

The HSOPS was created by the AHRQ, consisting of a self-administered survey including 42 items formulated as closed questions and used to calculate composite scores for 12 dimensions of safety culture [8]. In turn, the SAQ is commonly used to assess healthcare workers' perceptions of patient safety-related attitudes in various clinical areas and healthcare settings. The survey comprises six factors: teamwork climate, safety climate, job satisfaction, perceptions of management, working conditions, and stress recognition. The SAQ in its original generic form includes 60 items, 30 of which are core items [2].

Etchegaray and Thomas [9] studied the reliability and predictive validity of two patient safety culture surveys - the HSOPS and SAQ - when administered to the same group of respondents. They concluded that that both these tools had similar reliability and predictive validity, and that the researchers and practitioners should consider survey length, content, sensitivity to change, and the ability to compare when selecting a patient safety culture survey [9].

There are also other tools such as the Patient Safety Climate in Healthcare Organisations (PSCHO), the Canadian Patient Safety Climate Scale (Can-PSC), and the Safety Organising Scale (SOS). All the abovementioned tools used Likert scales with length ranging between 9 and 60 items of the questionnaire with a total of 141 items distributed under 36 climate di- mensions. Each tool covered between 1 (e.g. SOS) and 12 reported dimensions (e.g. HSOPS) [10].

The modern model of patient care consists of continuous advancement of medical knowledge, the pursuit of professionalism, and the application of world standards to ensure patient safety in the hospital. The unacquaintance of these issues can result in additional patient suffering, adverse events, and a decrease in the quality of provided medical services. At any stage of hospitalisation, i.e. from the time the patient is admitted to the hospital to the time of their discharge, adverse events may occur. An adverse event in healthcare is understood to be any unintended or unexpected event that could have caused harm, or did cause harm, to one or more patients receiving healthcare [11].

In the elaborated complexity and specificity of medical services it is not possible to completely eliminate adverse events; however, they can be prevented successfully. Medical errors are an inevitable part of the healthcare system and a substantial threat to patient safety [12]. Medication errors are the most common medical errors, which can occur as inappropriate use of medicine in each one of the medicine prescription stages for patients. Research has shown that among 44,000 to 98,000 deaths due to medical errors, as many as 7,000 were due to medication errors [13]. Medication error is a global issue, of which $5 \%$ are deadly and almost 50\% are preventable [14]. Nurses' interception of $86 \%$ of the medication errors was presented in a descriptive cross-sectional study conducted in Taiwan [15]. Also, studies showed a rate of serious patient injuries due to medication errors from $1 \%$ to $2 \%$, to $29 \%$, and as high as $51.8 \%$, and an estimated $30.5 \%$ of deaths per year in the USA [16]. It was reported that proportion of medication error among nurses can reach up to almost $60 \%$ and it is generally higher among female than male nurses [16].

One of the ways to prevent medical errors is to monitor adverse events using a patient safety monitoring system. Developing a registration system and an internal catalogue of adverse events, which should consider the threats of personnel, equipment, medications, and procedures, is a first step towards protecting the patient from lapses and errors. Due to the increasing requirements on healthcare quality and high expectations of patients, safety becomes an important component during diagnostic, therapeutic, nursing, and rehabilitation procedures. In the diagnostic process of patients, it is dangerous to commit laboratory errors that include delayed delivery, improper storage and transport of the samples after their collection, and incorrectly described samples and/or referral. Laboratory errors such as equipment breakdown, sample destruction during development, loss of sample identity at each stage of the testing, 
withdrawal of reagents by the company, and delayed delivery of the result by the provider may result in a false result of the test.

Radiological examinations play an important role in patient diagnosis. Errors in patient preparation for ultrasound examination, magnetic resonance imaging (MRI), computed tomography (CT), complications during examinations and post-diagnostic complications are treated as a group of undesirable events, of which the consequences may be the wrong diagnosis or therapy of the patient.

Sammer et al. [17] identified seven factors within patient safety culture: leadership, teamwork, evidence-based, communication, learning, just, and patient-centred.

About leadership, it has been stated that leaders recognise that healthcare is a high-risk environment. Therefore, it is necessary to combine financial and human resources. Teamwork was described as a collective feeling of collaboration to achieve a higher goal. Evidence-based, according to Sammer et al. [17], is patient care practices based on evidence. Standardisation serves to reduce the possibility of different outcomes. Processes need to be designed to achieve high dependability. The fourth subscale, communication, is the situation in which every employee has and feels the duty and responsibility to advocate for the patient [17].

The learning subscale is when the occurrence of the hospital improves its procedures as a direct result of first-hand experience. Learning is valued among all staff, including the medical staff. The subscale just is an environment that understands the detriment of assigning, blaming an individual when errors occur, and instead treating errors as a failure of policy, although it does not take issue with punishing individuals when individual errors occur. Patient-centred was defined by Sammer et al. [17] as "[...] focused on the patient and family. The patient takes an active part in his/her own care and acts as a mediator between the hospital and the community" [17].

\section{NURSE MANAGERS' COMPETENCIES}

One of the goals of this review was to identify the managers' competencies that affect patient safety. It seems that strong leadership leads to parity in the patient safety environment. In the last decade, the development and knowledge management of nursing has been examined from the perspective of administering EBP.

Sandström et al. [18] argued that leadership and organisational culture carry a foundational importance on the application of EBP. Their findings also indicate that leadership cannot be studied in isolation or without being clearly defined. Future research on this subject must consider the difficulty of study- ing organisational factors, which are constantly being challenged by developments in knowledge and changes in the use of resources as well as changes caused by political trends.

According to Kvist et al. [19], the majority of nurses $(62 \%)$ had positive perceptions towards developing nursing using research knowledge. Over $40 \%$ of them felt that the staff in their units did not have knowledge about evidence-based medicine (EBM). Nearly half (45\%) of nurse leaders appreciated the development of EBP as an important part of their leadership style. Most of the nurse leaders (88\%) disagreed that their staff had sufficient time at their work to read research from their own disciplines. $29 \%$ of nurse leaders did not encourage their staff to explore the latest research in their own area. Half of nurse leaders (50\%) felt that they were not able to make it possible for their staff to perform EBP by giving time to search for evidence, and 38\% disagreed that when choosing new staff, their research experience was not the first priority.

Some studies, such as one by Wilkinson et al. [20], indicate that when it comes to accommodating EBP some leaders are passive and not sufficiently committed to adopting it. The results of this study highlight the complexity of EBP implementation, which is determined by a number of contextual factors. One of the proposals that shaped the analysis of the data was that evidence-based nursing is likely to be more relevant in organisations where directors and nurse managers are leaders and support nursing staff [20].

Omoike et al. [21] showed that educational training is useful in improving nursing competences in their role at the system level. The Leadership Survey identified differences in nurse manager ratings between importance and competence. The pre-program relationship between how respondents rated the level of overall importance (4.33) in any activity and their perceived competence (3.47) in that activity did not match, resulting in significant differences in $11(92 \%)$ of the 12 subscales. Participants perceived themselves as more competent post program in 11 of the 12 subscales. Although there is recognition of the importance of fostering the development of staff competency among leaders, nearly $50 \%$ of them believe that they are incapable of managing competency [21].

It has even been found that some nurses are insecure in their knowledge, which affects their ability to use it at work [22]. This is echoed by WHO'S THE SOURCE, which also found that some leaders view their knowledge management skills as inadequate.

According to Phillips et al. [23] and their findings from a systematic review (MEDLINE, Academic Search Premier, ERIC, CINAHL, Scopus, Embase, Informit health, Cochrane Library and Web of Science databases), there is still scarce evidence detailing the 
effectiveness of interventions encouraging evidencebased practice. Additionally, interventions have not been reported in enough detail to repeat and compare these studies. The authors concluded that determination of the essential components of the specification of educational interventions aimed at facilitating the acquiring of fundamental knowledge and skills in the EBP remains to be established.

Lunden et al. [24] pointed out that "leadership models", "leader's traits", and "leader's competence" are facilitating factors related to leadership. Competency among employees has been widely found to be improved through magnet hospital status and transformational leadership in relation to leadership models [25-27]. Magnet hospital status refers to an institution that has successfully attracted and retained nurses. Salmond et al. [28] highlighted that magnet hospitals cherish the continual improvement and maintenance of high quality competency at every level of organisation.

It is worth emphasising that the magnet hospital designation was formalised with the American Nurses Credentialing Centre's (ANCC) Magnet Recognition Program in the 1990s [29]. This program focuses on nursing excellence and targets a series of benchmarks: "transformational leadership, a structure that empowers staff, an established professional nursing practice model, support for knowledge generation and application, and robust quality improvement mechanisms" [30]. The hospitals that achieve magnet recognition seem to improve in terms of nurses' performance (i.e. job satisfaction, burnout, intention to leave), patients' performance (i.e. mortality, failure to rescue), and performance measures (i.e. value-based purchases) to a greater extent than other hospitals that do not have the same results [31].

\section{IMPORTANT FACTORS}

Knowledge management promoting leadership consists of the leader's dedication to their task and the accuracy, duty, dependability, and security of their role [27, 32]. Other connected aspects include long-term planning and effective leadership style and cooperation [27, 33]. Leader's competency can be measured by a profound education and evolved by continual development and structuring [21, 27, 34]. Codier et al. [35] and Brinkert [36] underlined that emotional intelligence and general leadership skills can be honed by peer and conflict coaching.

Currently in clinical settings, evidence obtained through research does not reveal any workable solutions to govern knowledge management. In light of this, practical interventions should be evaluated, developed, and utilised so as to better administer knowledge management. This is especially true in contemporary, challenging work environments with a lack of time for further professional development due to an increasing workload. This we should call a rationing of care.

\section{CARE RATIONING}

The factors influencing the quality of nursing practice are the adopted standards of care, the number of employed nurses and midwives, as well as their qualifications, psychophysical determinants, and motivations. To provide an adequate level of care, it is necessary to establish and adhere to the accepted standards of the medical staff employment. In the era of poorly understood savings in the health care system and absence of nurses, restructuring of nursing staff balances on the border of safety. This is evidenced, among other by the experience of physical injury of a patient during hospitalisation including: falls, trips, sprains, burns, and patients falling out of bed. The danger is also present due to the use of equipment for lifting and moving the patients. Its users should carefully read the manufacturer's instructions to avoid injuries related to the lack of ability to properly use such equipment. Obeying the principles of good practice is the responsibility of the medical staff to ensure proper patient care. Minimum staff employment standards do not guarantee the desirable level of quality of medical services and the implementation of adopted standards of conduct.

In the currently changing conditions, in which nurses are lacking around the world, we can already talk about nursing care deficiencies and the rationing of nursing care. This problem probably has not been solved in this decade. Nursing is a very difficult profession that requires a lot of sacrifices, and young people do not want to study in this field, which will result in the aging of nurses currently working and the seriously increasing deficiencies of new nursing entrants. The concept of rationing of care has already arisen. Papastavrou et al. [37] refer to rationing of care as a phenomenon related to the issue of fair distribution of resources.

Rationing of care or missed care can be categorised as errors of omission, which means that some nursing care is delayed, only partially completed, or not completed at all. Kalisch et al. [38] developed a conceptual model of missed nursing care, in which the structural factors contributing to missed nursing care include: labour resources, material resources, and teamwork and communication. If any of these elements are lacking, nurses are obliged to prioritise their tasks, and at this point nursing care might be delayed or omitted.

The problem of missed care is a well-established concept in the nursing literature. Evidence of missed care exists - the problem has been studied in a number of countries [38-40]. Ausserhofer et al. [39], in 
their large multi-country cross-sectional study, collected data from 33,659 nurses in 488 European hospitals across 12 countries. They reported that the most frequent missed nursing care activities included 'comfort/talk with patients' (53\%), 'developing or updating nursing care plans/care pathways' (42\%), and 'educating patients and families' (41\%). In hospitals with more favourable work environments, lower patient-to-nurse ratios, and lower proportions of nurses carrying out non-nursing tasks frequently, fewer nurses reported nursing care left undone. Also, Ball et al. [40] conducted a cross-sectional survey ( $n=2917$ registered nurses) working in National Health Service hospitals in England. They showed that most nurses (86\%) reported that one or more care activity had been missed due to lack of time on their last shift. Missed nursing care was most frequent in the case of: comforting or talking with patients (66\%), educating patients (52\%), and developing/updating nursing care plans (47\%). A mean of 7.8 activities per shift were left undone on wards rated as 'failing' on patient safety, compared with 2.4 activities on wards where patient safety was rated as 'excellent'.

Recently, Jones et al. [41] published an integrated review on the subject of missed care and found that three conceptualisations of omitted care have been developed by different research teams: care left undone [42]; implicit rationing of care [43]; and missed care $[38,44]$. All proposed conceptualisations look to examine the relationships between organisational factors such as staffing levels, and client and staffing outcomes. These concepts highlight the problem inherent in identifying which nursing tasks and activities are omitted when there is a lack of time and resources, making the provision of necessary care difficult $[39,41]$.

Jones et al. [41] performed a literature review using CINAHL and MEDLINE, which identified 1828 articles, of which 54 articles were reviewed using 14 self-report instruments for measuring unfinished care. They observed that most nursing personnel (55-98\%) reported leaving one or more activities unfinished (mean 2-21). The reported estimates of the prevalence of unfinished care increased as the size of the inventory increased and as the recall period increased from one to seven shifts. High betweencountry and within-country variation in unfinished care was demonstrated (12 European countries, 488 hospitals, $n=33,659)$. Based on composite scores of the TU-13, five countries demonstrated higher reported missed care than the European average (3.6): Ireland (3.8), England (4.0), Belgium (4.1), Germany (4.7), and Greece (5.8).

Currently, we have tools with which we can measure missed nursing care, but ongoing research is required on the use of such tools. Missed care can be measured by the "Tasks left undone" scale developed by Lucero et al. [45]. This 12-item scale asks nurses to identify patient care activities on their last shift that they considered necessary but which were left undone. Another measure is the Basel Extent of Rationing of Nursing Care (BERNCA) questionnaire developed by Schubert et al. [43], which allows measurement of the levels of implicit rationing of nursing care in acute care hospitals. Kalisch and Williams [46] proposed a MISSCARE survey to measure missed nursing care, and to identify the reasons for missed nursing care. It is necessary to understand that the most important predictors of missed nursing care are staffing levels and teamwork. Nowadays, as we face a global lack of nurses, missed care is a common threat, and it should, therefore, garner greater recognition because it can manifest as a potentially dangerous medical error. It has already been pointed out that the problem of missed nursing care deserves special attention [47]. Further studies using validated tools are advisable.

Several tools have recently been developed to assess the rationing of nursing care. The best known, the BERNCA instrument, was created by Schubert et al. [48] and allows us to measure the level of rationing of care in hospitals. In turn, Kalisch and Williams [44] proposed a questionnaire called the Missed Nursing Care (MISSCARE) Survey, which in addition to omitting care also assesses its causes. It should be noted that until 2018 there was no Polish tool to evaluate the level of rationing of nursing care. However, since then, three tools have been adapted by the authors of this paper: the BERNCA in 2019 [49], the Perceived Implicit Rationing of Nursing Care (PIRNCA) in 2020 [50], and the Neonatal Extent of Work Rationing Instrument (NEWRI) in 2020 [51].

\section{CONCLUSIONS}

To summarise the issue of patient safety in hospitals and nurse managers' competencies relating to rationing of care, it can be concluded that it is necessary to work out and create the right system solution that challenges the modern health care system.

\section{Disclosure}

The authors declare no conflict of interest.

\section{References}

1. Health and Safety Commission Advisory Committee on the Safety of Nuclear Installations. Advisory Committee on the Safety of Nuclear Installations (ACSNI) study group on human factors. 1993. http://inis.iaea.org/Search/search. aspx?orig_q=RN:25003245 (accessed: 24 July 2017).

2. Sexton JB, Helmreich RL, Neilands TB, et al. The Safety Attitudes Questionnaire: psychometric properties, benchmarking data, and emerging research. BMC Health Serv Res 2006; 6: 44. 
3. Singer S, Meterko M, Baker L, et al. Workforce Perceptions of Hospital Safety Culture: Development and Validation of the Patient Safety Climate in Healthcare Organizations Survey. Health Serv Res 2007; 42: 1999-2021.

4. Sorra J, Gray L, Streagle S, et al. AHRQ Hospital Survey on Patient Safety Culture: User's Guide. Agency for Healthcare Research and Quality (AHRQ) Publication No. 15-0049-EF. Rockville, MD: USA 2016. https://www.ahrq.gov/sites/ default/files/wysiwyg/professionals/quality-patient-safety/ patientsafetyculture/hospital/userguide/hospcult.pdf

5. World Health Organisation. Patient Safety: Making health care safer. World Health Organization. 2007. http://www. who.int/patientsafety/publications/patient-safety-makinghealth-care-safer/en/ (accessed: 24 July 2017).

6. Waterson P, Carman EM, Manser T, Hammer A. Hospital Survey on Patient Safety Culture (HSPSC): a systematic review of the psychometric properties of 62 international studies. BMJ Open 2019; 9: e026896.

7. Okuyama JHH, Galvao TF, Silva MT. Healthcare professional's perception of patient safety measured by the hospital survey on patient safety culture: a systematic review and meta-analysis. Sci World J 2018; 2018: 9156301.

8. Boussat B, Kamalanavin K, François P. The contribution of open comments to understanding the results from the Hospital Survey on Patient Safety Culture (HSOPS): A qualitative study. PLoS One 2018; 13. e0196089.

9. Etchegaray JM, Thomas EJ. Comparing two safety culture surveys: safety attitudes questionnaire and hospital survey on patient safety. BMJ Qual Saf 2012; 21: 490-498.

10. Alsalem G, Bowie P, Morrison J. Assessing safety climate in acute hospital settings: a systematic review of the adequacy of the psychometric properties of survey measurement tools. BMC Health Serv Res 2018; 18: 353.

11. Council of Europe. Appendix to Recommendation of the Committee of Ministers to member states on management of patient safety and prevention of adverse events in health care CM/Del/Dec(2006)965.

12. Fathi A, Hajizadeh M, Moradi K, et al. Medication errors among nurses in teaching hospitals in the west of Iran: what we need to know about prevalence, types, and barriers to reporting. Epidemiol Health 2017; 39: e2017022.

13. Gorgich EAC, Barfroshan S, Ghoreishi G, Yaghoobi M. Investigating the causes of medication errors and strategies to prevention of them from nurses and nursing student viewpoint. Glob J Health Sci 2016; 8: 220-227.

14. Nuckols TK, Bell DS, Liu H, et al. Rates and types of events reported to established incident reporting systems in two US hospitals. Qual Saf Health Care 2007; 16: 164-168.

15. Chiang HY, Pepper GA. Barriers to nurses' reporting of medication administration errors in Taiwan. J Nurs Scholarsh 2006; 38: 392-399.

16. Jember A, Hailu M, Messele A, et al. Proportion of medication error reporting and associated factors among nurses: a cross sectional study. BMC Nurs 2018; 17. doi: 10.1186/ s12912-018-0280-4.

17. Sammer CE, Lykens K, Singh KP, et al. What is patient safety culture? A review of the literature. J Nurs Scholarsh 2010; 42: 156-165.

18. Sandström B, Borglin G, Nilsson R, Willman A. Promoting the implementation of evidence-based practice: a literature review focusing on the role of nursing leadership. Worldviews Evid Based Nurs 2011; 8: 212-223.
19. Kvist T, Tähkä K, Ruotsalainen M, et al. The impact of nursing leadership training on evidence-based leadership and practice. J Nurs Care 2014; 3. doi: 10.4172/2167-1168.1000181.

20. Wilkinson JE, Nutley SM, Davies HTO. An exploration of the roles of nurse managers in evidence-based practice implementation. Worldviews Evid Based Nurs 2011; 8: 236-246.

21. Omoike O, Stratton KM, Brooks BA, et al. Advancing nursing leadership: a model for program implementation and measurement. Nurs Adm Q 2011; 35: 323-332.

22. Saunders $H$, Vehviläinen-Julkunen $K$. The state of readiness for evidence-based practice among nurses: An integrative review. Int J Nurs Stud 2016; 56: 128-140.

23. Phillips AC, Lewis LK, McEvoy MP, et al. A systematic review of how studies describe educational interventions for evidencebased practice: stage 1 of the development of a reporting guideline. BMC Med Educ 2014; 14: 152.

24. Lunden A, Teräs M, Kvist T, Häggman-Laitila A. A systematic review of factors influencing knowledge management and the nurse leaders' role. J Nurs Manag 2017; 25: 407-420.

25. Spicer JG, Guo Y, Liu H, et al. Importance of role competencies for Chinese directors of nursing based on the forces of magnetism. J Nurs Manag 2011; 19: 153-159.

26. Strickland RJ, O'Leary-Kelley C. Clinical nurse educators' perceptions of research utilization: barriers and facilitators to change. J Nurses Staff Dev 2009; 25: 164-171; quiz 172-173.

27. Leggat SG, Balding C. Achieving organisational competence for clinical leadership: the role of high performance work systems. J Health Organ Manag 2013; 27: 312-329.

28. Salmond SW, Begley R, Brennan J, Saimbert MK. A comprehensive systematic review of evidence on determining the impact of Magnet designation on nursing and patient outcomes: is the investment worth it? JBI Libr Syst Rev 2009; 7: 1119-1178.

29. McHugh MD, Kelly LA, Smith HL, et al. Lower mortality in Magnet hospitals. Med Care 2013; 51: 382-388.

30. Keeping Patients Safe: Transforming the Work Environment of Nurses. Transformational Leadership and Evidence-Based Management. National Academies Press (US); 2004. https:// www.ncbi.nlm.nih.gov/books/NBK216194/ (accessed: 21 April 2020).

31. Lasater KB, Richards MR, Dandapani NB, et al. Magnet hospital recognition in hospital systems over time. Health Care Manage Rev 2019; 44: 19-29.

32. Chen JC, Silverthorne C, Hung JY. Organization communication, job stress, organizational commitment, and job performance of accounting professionals in Taiwan and America. Leadersh Organ Dev J 2006; 27: 242-249.

33. Romain-Glassey N, Ninane F, de Puy J, et al. The emergence of forensic nursing and advanced nursing practice in Switzerland: an innovative case study consultation. J Forensic Nurs 2014; 10: 144-152.

34. Hsu HY, Lee LL, Fu CY, et al. Evaluation of a leadership orientation program in Taiwan: preceptorship and leader competencies of the new nurse manager. Nurse Educ Today 2011; 31: 809-814. 